Acta Univ. Sapientiae Informatica 13, 1 (2021) 21-38

DOI: $10.2478 /$ ausi-2021-0002

\title{
Confluence number of certain derivative graphs
}

\author{
Johan KOK \\ Independent Mathematics Researcher, \\ City of Tshwane, South Africa \& \\ Visiting Faculty at CHRIST (Deemed to \\ be a University), Bangalore, India \\ email: jacotype@gmail.com
}

\author{
Joseph SHINY \\ Mathematics Research Center, \\ Mary Matha Arts and Science College \\ Mananthavady, Kerala, India \\ email: shinyjoseph314@gmail.com
}

\begin{abstract}
This paper furthers the study on the confluence number of a graph. In particular results for certain derivative graphs such as the line graph of trees, cactus graphs, linear Jaco graphs and novel graph operations are reported.
\end{abstract}

\section{Introduction}

Concepts, notation and graph parameters without formal definitions can be clarified in $[3,4,12]$. Unless stated otherwise, graphs will be finite, undirected and non-complete, connected simple graphs. A shortest path having end vertices $u$ and $v$ is denoted by $u-v_{(\text {in } G)}$. If $d_{G}(u, v) \geq 2$ then a vertex $w$ on $u-v_{(\text {in } G)}, w \neq u, w \neq v$ is called an internal vertex on $u-v_{(\text {in } G)}$. When the context is clear the notation such as $d_{G}(u, v), \operatorname{deg}_{G}(v)$ can be abbreviated to $\mathrm{d}(u, v), \operatorname{deg}(v)$ and so on.

The notion of a confluence set (a subset of vertices) of a graph $G$ was introduced in [11]. For a non-complete graph $\mathrm{G}$ a non-empty subset $\mathcal{X} \subseteq \mathrm{V}(\mathrm{G})$ is said to be a confluence set if for every unordered pair $\{u, v\}$ of distinct vertices

Computing Classification System 1998: G.2.2

Mathematics Subject Classification 2010: 05C07, $05 \mathrm{C} 12$

Key words and phrases: Confluence set, minimum confluence set, confluence number. 
(if such exist) in $\mathrm{V}(\mathrm{G}) \backslash \mathcal{X}$ for which $\mathrm{d}_{\mathrm{G}}(\mathrm{u}, v) \geq 2$ there exists at least one $u-v_{(\text {in } \mathrm{G})}$ with at least one internal vertex $\boldsymbol{w} \in \mathcal{X}$. Also any vertex $u \in \mathcal{X}$ is called a confluence vertex of $\mathrm{G}$. A minimal confluence set $\mathcal{X}$ has no proper subset which is a confluence set of $\mathrm{G}$. The cardinality of a minimum confluence set denoted by $\mathcal{C}$ (also called confluence set $\mathcal{C}$ when context is clear) is called the confluence number of $\mathrm{G}$ and is denoted by $\zeta(\mathrm{G})$. To distinguish between different graphs the notation $\mathcal{C}_{\mathrm{G}}$ may be used for a minimum confluence set of G.

\section{Preliminaries}

Recall that the line graph $\mathrm{L}(\mathrm{G})$ of graph $\mathrm{G}$ is obtained by letting $\mathrm{V}(\mathrm{L}(\mathrm{G}))=$ $\left\{e_{i}^{\prime}: e_{i}^{\prime}\right.$ a vertex representation of the edge $e_{i}$ iff $\left.e_{i} \in E(G)\right\}$ and $E(L(G))=$ $\left\{e_{i}^{\prime} e_{j}^{\prime}\right.$ : iff $e_{i}, e_{j}$ share a common end-vertex $\}$. A number of known results related to a line graph and which are important for this paper are recalled throughout.

Theorem 1 [10] For a path $\mathrm{P}_{\mathrm{n}}, \mathrm{n} \geq 2, \mathrm{~L}\left(\mathrm{P}_{\mathrm{n}}\right)=\mathrm{P}_{\mathrm{n}-1}$.

A corollary is immediate from Theorem 1.

Corollary 2 For a path $\mathrm{P}_{\mathrm{n}}, \mathrm{n} \geq 2$ :

$$
\zeta\left(\mathrm{L}\left(\mathrm{P}_{\mathrm{n}}\right)\right)= \begin{cases}0, & \text { if } \mathrm{n}=2 \\ \left\lfloor\frac{\mathrm{n}-1}{3}\right\rfloor, & \text { if } \mathrm{n} \geq 3 .\end{cases}
$$

Proof. The result follows from Theorem 1 read together with Theorem 5 in [11] i.e.:

$$
\zeta\left(P_{n}\right)= \begin{cases}0, & \text { if } n=1 \text { or } 2 \\ \left\lfloor\frac{n}{3}\right\rfloor, & \text { if } n \geq 3\end{cases}
$$

A subsequent corollary is trivial.

Corollary 3 For a path $\mathrm{P}_{\mathrm{n}}, \mathrm{n} \geq 2$ it follows that $\zeta\left(\mathrm{P}_{\mathrm{n}}\right)>\zeta\left(\mathrm{L}\left(\mathrm{P}_{\mathrm{n}}\right)\right)$ if $\mathrm{n}=3 \mathrm{t}$, $\mathrm{t}=1,2,3, \ldots$ Else, $\zeta\left(\mathrm{P}_{\mathrm{n}}\right)=\zeta\left(\mathrm{L}\left(\mathrm{P}_{\mathrm{n}}\right)\right)$.

Theorem 4 A path $\mathrm{P}_{\mathrm{n}}$ has a unique minimum confluence set $\mathcal{C}_{\mathrm{P}_{\mathrm{n}}}$ if and only if $\mathrm{n}=5+3 i, \mathrm{i}=0,1,2, \ldots$

Proof. Let path $P_{n}$ be on the consecutive vertices $v_{1}, v_{2}, v_{3}, \ldots, v_{n}$. For $P_{5}$ it is trivial that $\left\{v_{3}\right\}$ is the unique minimum confluence set. 
It is easy to verify that if $v_{i}, v_{j} \in \mathcal{C}_{P_{n}}$ then $d\left(v_{i}, v_{j}\right) \leq 3$. By ensuring $\mathrm{d}\left(v_{i}, v_{j}\right)=$ 3 to a maximum results in a minimum confluence set for $P_{n}$. In particular for $\mathrm{n}=5+3 i, i=1,2,3, \ldots$ the selection of a minimum confluence set yields a unique minimum confluence set for $P_{n}$. This settles the "if".

The converse follows inherently from the minimum confluence set selection procedure and the well-defined value $n=5+3 i, i=0,1,2, \ldots$ in the first part.

Counter example. For illustrative purpose consider $P_{6}=v_{1} v_{2} v_{3} v_{4} v_{5} v_{6}$. It is easy to see that amongst others, the sets $\left\{v_{3}, v_{4}\right\},\left\{v_{3}, v_{5}\right\}$ and $\left\{v_{3}, v_{6}\right\}$ are non-unique minimum confluence sets of $\mathrm{P}_{6}, 6 \neq 5+3 i$ for any $i$.

Theorem 5 [10] A graph $\mathrm{G}$ is isomorphic to its line graph if and only if $\mathrm{G}$ is a cycle $\mathrm{C}_{\mathrm{n}}, \mathrm{n} \geq 3$.

Theorem 5 implies that $\mathrm{C}_{n} \cong \mathrm{L}\left(\mathrm{C}_{n}\right) \cong \mathrm{L}\left(\mathrm{L}\left(\mathrm{C}_{n}\right)\right) \cong \cdots \cong \mathrm{L}\left(\mathrm{L} \cdots \mathrm{L}\left(\mathrm{C}_{n}\right)\right)$. Therefore, $\zeta\left(C_{n}\right)=\zeta\left(L\left(C_{n}\right)\right), n \geq 3$. With only a change in notation we adopt the result from [11].

Theorem 6 For a cycle $\mathrm{C}_{\mathrm{n}}, \mathrm{n} \geq 3$ :

$$
\zeta\left(\mathrm{L}\left(\mathrm{C}_{\mathrm{n}}\right)\right)= \begin{cases}0, & \text { if } \mathrm{n}=3 \\ 1, & \text { if } \mathrm{n}=4 \\ \left\lceil\frac{\mathrm{n}}{3}\right\rceil, & \text { if } \mathrm{n} \geq 5 .\end{cases}
$$

The next lemma is a consequence of the definition of a minimum confluence set.

Lemma 7 Consider a graph $\mathrm{G}$ with at least two vertices $\mathrm{u}, \boldsymbol{v}, \mathrm{d}_{\mathrm{G}}(\mathrm{u}, \boldsymbol{v})=2$. Amongst all possible $\left.\mathrm{u}-\boldsymbol{v}_{(\mathrm{in})} \mathrm{G}\right)$ there exists at least one such path say, uwv such that $\mathrm{u}$ or $\boldsymbol{w}$ or $\boldsymbol{v}$ an element $(s)$ of $\mathcal{C}_{\mathrm{G}}$.

A vertex $v$ to which a leaf (pendent) vertex $u$ is attached is called the pre-leaf of $u$ or simply, pre-leaf $v$.

Lemma 8 For any graph $\mathrm{G}$ of order $\mathrm{n} \geq 3$ which has a leaf $\mathrm{u}$ there exists a confluence set $\mathcal{C}$ (a minimum) such that $\mathfrak{u} \notin \mathcal{C}$.

Proof. Let a leaf $u \in \mathcal{C}$ and let $v$ be the pre-leaf vertex of $u$. Clearly if both $u, v \in \mathcal{C}$ then $\mathcal{C}$ is not minimal thus not minimum. Therefore, $v \notin \mathcal{C}$ hence $(\mathcal{C}-u) \cup\{v\}$ remains a minimum confluence set of $\mathrm{T}$. This suffices to settle the result. 
Lemma 8 implies that since a pendent vertex cannot be an internal vertex on a path it need not by necessity be an element of a minimum confluence set $\mathcal{C}$ of any graph.

Let the pendent degree of vertex $u \in V(G)$ denoted by $\operatorname{deg}_{p}(u)$ be the number of pendent vertices adjacent to $u$. The vertex set $V(G)$ can be partitioned into $X_{1}=\left\{u: \operatorname{deg}_{p}(u)=0\right\}, X_{2}=\left\{v: \operatorname{deg}_{p}(v)=1\right\}$ and $X_{3}=\left\{w: \operatorname{deg}_{p}(w) \geq 2\right\}$. A trivial lower bound establishes i.e. $\zeta(G) \geq\left|X_{3}\right|$. Put differently, there exists a minimum confluence set $\mathcal{C}$ of $T$ such that $X_{3} \subseteq \mathcal{C}$. The aforesaid is true because the unique shortest path between any two pendent vertices sharing a common pre-leaf $w$ permits $w \in \mathcal{C}$. For a tree $T$ this lower bound can be useful and equality can hold. For example for a star, $S_{1, n} \cong K_{1, n}$. Let $X_{1}^{\prime}=\{$ leafs of $T\}$. Note that $X_{1}^{\prime} \subseteq X_{1}$.

Proposition 9 If for a tree $\mathrm{T}$ the vertex set partition is such that $\mathrm{V}(\mathrm{T})=$ $\mathrm{X}_{1}^{\prime} \cup \mathrm{X}_{3}$ then $\zeta(\mathrm{T})=\left|\mathrm{X}_{3}\right|$.

Proof. Since $V(T)=X_{1}^{\prime} \cup X_{3}$ the vertex set $V(T)$ consists of only leafs and pre-leafs. Furthermore, each pre-leaf $u$ has at least two leafs say, $v, w$. There exist a unique $v-w_{(\text {in } T)}$ with the unique internal vertex $u$ so it follows by necessity that $u \in \mathcal{C}$ so, $\zeta(T) \geq\left|X_{3}\right|$. By Lemma 8 a leaf is not by necessity in $\mathcal{C}$ so $\zeta(T) \leq\left|X_{3}\right|$. Therefore $\zeta(T)=\left|X_{3}\right|$.

\section{$2.1 \quad$ Heuristic method to obtain $\zeta(T)$}

The path $P_{n}$ is called a n-path. For paths $P$ and $Q$ we define $P \cap Q=$ $V(P) \cap V(Q)$. For different paths and not necessarily of different order, $P_{n_{1}}$, $P_{n_{2}}, P_{n_{3}}, \ldots, P_{n_{k}}$ we define $\bigcap_{i=1}^{k} P_{n_{i}}=\left(\left(\left(P_{n_{1}} \cap P_{n_{2}}\right) \cap P_{n_{3}}\right) \cap \cdots \cap P_{n_{k}}\right)$.

Heuristic A. For a vertex labeled tree $T$ of order $n \geq 3$ do:

Step 1A. Let $V(T)=\left\{v_{1}, v_{2}, v_{3}, \ldots, v_{n}\right.$ with $v_{n}$ a leaf $\}$. Minimizing by isomorphism let $\mathfrak{P}$ be maximal with $\mathfrak{P}=\left\{\right.$ all 3-paths $\left.v_{1} v_{i} v_{j},\right\} \cup\{$ all 3-paths $\left.v_{2} v_{k} v_{l},\right\} \cup \cdots \cup\left\{\right.$ all 3-paths $\left.v_{n-1} v_{m} v_{q}\right\}$.

Step 2A: Consider the vertex subset of $V(T)$ i.e. $X_{3}=\left\{w: \operatorname{deg}_{p}(w) \geq 2\right\}$.

Step 3A: For all $x \in X_{3}$ remove the 3-paths in $\mathfrak{P}$ which contains vertex $x$ to obtain $\mathfrak{P}^{\prime}$.

Step 4A. Partition $\mathfrak{P}^{\prime}$ into a minimum partition of 3-path subsets such that, within a 3-path subset all 3-paths share at least a common vertex except for singleton subsets. Denote this partition by $\mathcal{P}\left(\mathfrak{P}^{\prime}\right)$.

Step 5A. Let the number of 3-path subsets in $\mathcal{P}\left(\mathfrak{P}^{\prime}\right)$ be $\ell$. Then $\zeta(T)=\ell+\left|X_{3}\right|$. 
Furthermore, for a singleton 3-path subset in $\mathcal{P}\left(\mathfrak{P}^{\prime}\right)$ any one vertex on the 3path is permitted in $\mathcal{C}_{\mathrm{T}}$. For 3-path subsets with two or more 3-paths which share two common vertices, any one of the two vertices is permitted in $\mathcal{C}_{\text {T }}$. For 3-path subsets with two or more 3-paths which share one common vertex, only the common vertex is permitted in $\mathcal{C}_{\mathrm{T}}$.

Note that prescribing $v_{n}$ to be a leaf is only a matter of convenience. Furthermore, besides obtaining the confluence number the heuristic yields a valid minimum confluence set. In real world applications this additional result can be worthy.

Theorem 10 Heuristic $A$ is valid.

Proof. Since the 3-path between any two vertices in $T$ is unique, every 3-path in Step $1 \mathrm{~A}$ is unique. Lemma 7 implies that no 3-path can exists without at least one vertex in $\mathcal{C}_{\mathrm{T}}$. Therefore a set $\mathcal{X}=\left\{v_{i}: v_{i}\right.$ a central vertex of a 3-path in $\mathfrak{P}\}$ is a confluence set of $\mathrm{T}$. Hence, the validity of the Heuristic A follows immediately from minimizing $\mathfrak{P}$ by isomorphism in Step $1 \mathrm{~A}$ and the minimization of partition $\mathfrak{P}^{\prime}$ in Step $4 \mathrm{~A}$ to yield $\mathcal{C}_{\mathrm{T}}$.

Applying Heuristic A to a general graph will yield a confluence set denoted by $\mathcal{X}_{h}$. It follows trivially that $\zeta(\mathrm{G}) \leq \mathcal{X}_{h}$. The advantage of the aforesaid approach for a real world application is that after a $\mathcal{X}_{h}$ has been obtained, $\mathcal{X}_{h}$ can be minimized to yield a valid minimum confluence set $\mathcal{C}_{\mathrm{G}}$.

Example: Consider the tree $T$ of order 12 in figure 1.

Step $1 \mathrm{~A}$. Minimizing by isomorphism the maximum set $\mathfrak{P}$ of 3 -paths (elements) is,

$\mathfrak{P}=\left\{v_{1} v_{3} v_{2}, v_{1} v_{3} v_{4}, v_{2} v_{3} v_{4}, v_{3} v_{4} v_{5}, v_{3} v_{4} v_{6}, v_{4} v_{6} v_{7}, v_{5} v_{4} v_{6}, v_{6} v_{7} v_{8}, v_{6} v_{7} v_{9}\right.$, $\left.v_{7} v_{9} v_{10}, v_{7} v_{9} v_{11}, v_{7} v_{9} v_{12}, v_{8} v_{7} v_{9}, v_{10} v_{9} v_{11}, v_{10} v_{9} v_{12}, v_{11} v_{9} v_{12}\right\}$

Step 2 A. $X_{3}=\left\{v_{3}, v_{9}\right\}$.

Step 3A. After removing all 3-paths which have vertices $v_{3}$ or $v_{9}$ the set $\mathfrak{P}^{\prime}=$ $\left\{v_{4} v_{6} v_{7}, v_{5} v_{4} v_{6}, v_{6} v_{7} v_{8}\right\}$ is obtained.

Step 4A. In respect of $\mathfrak{P}^{\prime}$ a minimum partition of 3-path subsets such that, within a 3-path subset all 3-paths share a single vertex is $\mathcal{P}\left(\mathfrak{P}^{\prime}\right)=\left\{\left\{v_{4} v_{6} v_{7}, v_{5} v_{4} v_{6}, v_{6} v_{7} v_{8}\right\}\right\}$. Therefore, $\ell=1$.

Step 5A. The result $\zeta(T)=1+2=3$. Moreover, a minimum confluence set is $\mathcal{C}_{\mathrm{T}}=\left\{v_{3}, v_{6}, v_{9}\right\}$. 


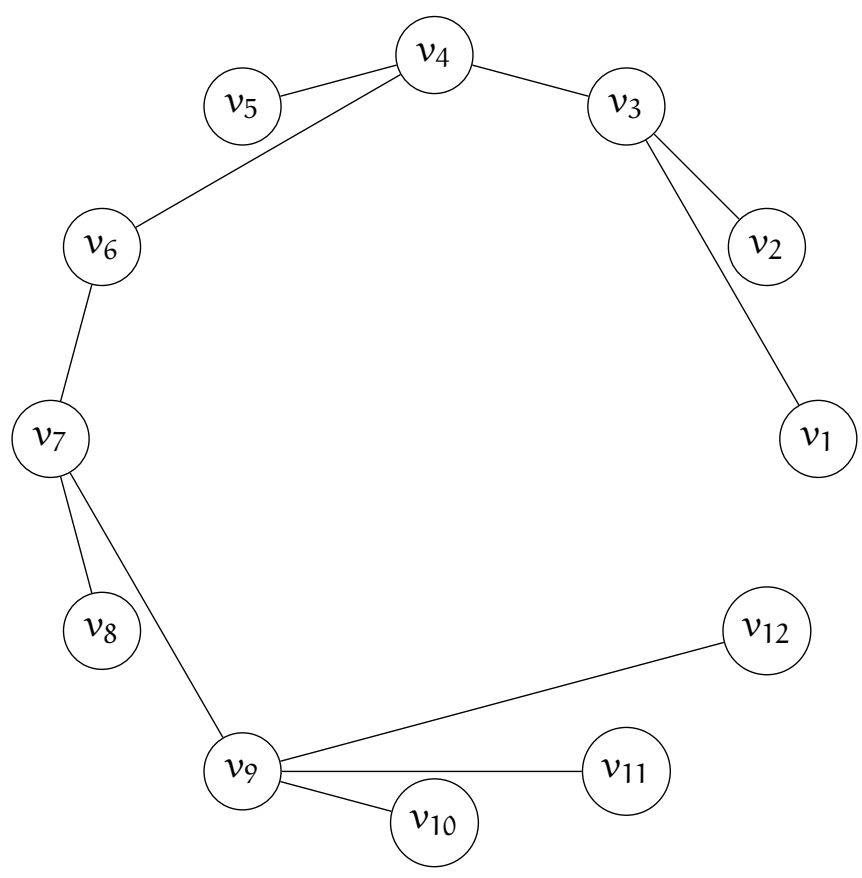

Figure 1: Tree $\mathrm{T}$ of order 12.

\section{$3 \quad$ Line graph of trees}

From an intersection graph perspective we recall an important definition.

Definition 11 Let $\mathfrak{C}$ be a non-empty set of non-empty subgraphs of $\mathrm{G}$. Then let each element (subgraph) in $\mathfrak{C}$ be represented by a unique vertex say $v_{i}$. Hence, $v_{i} \in \mathfrak{C}, \mathfrak{i}=1,2,3, \ldots,|\mathfrak{C}|$ has well-defined meaning. Define the derivative graph $\mathrm{G}(\mathfrak{C})$ on the vertex set $\mathfrak{C}$ with edge set $\mathrm{E}(\mathrm{G}(\mathfrak{C}))=\left\{v_{i} v_{j}\right.$ : if and only if $v_{i} \neq v_{j}$ and $v_{i}, v_{j}$ satisfy some adjacency condition $\}$.

Note that an adjacency condition can be a condition such as, conventional adjacency between vertices or edges incident with a common vertex. However, in an abstract sence, it could be subsets $X_{i} \subset V(G), i \geq 2$ such that $\bigcup X_{i}=V(G)$ and $X_{i} \cap X_{i}=\emptyset, i \neq j$. Applications in abstract algebra present all $i$

numerous innovative 'adjacency conditions'.

For a graph $G$ let $\mathfrak{C}=E(G)$. Then the line graph of $G$ denoted by $L(G)$ is defined by $\mathrm{L}(\mathrm{G})=\mathrm{G}(\mathfrak{C})$ with $\mathrm{V}(\mathrm{L}(\mathrm{G}))=\mathfrak{C}$ and $\mathrm{E}(\mathrm{L}(\mathrm{G}))=\left\{v_{i} v_{j}\right.$ : if and only if $v_{i}, v_{j}$ are adjacent in $\left.\mathrm{G}\right\}$. 
In the literature there exist different views on the distinction between block graphs and cactus graphs. This section will accept the view that a block graph is a graph whose blocks are cliques. Furthermore, in a block graph the intersection between any two distinct blocks are either empty or a cut vertex. Note that a tree $T$ is a block graph. See a good characterization of block graphs in [2]. A path $P_{n}, n \geq 3$ which has an end-vertex merged with a vertex of $G$ which is not a path itself and the other end-vertex of the path remains pendent is a beam of G.

For a graph $\mathrm{G} \not \mathrm{P}_{\mathrm{n}}, \mathrm{n} \geq 3$ the line graph $\mathrm{L}(\mathrm{G})$ typically has a combination of the structural elements (not necessarily all), (i) cliques of order $\geq 3$ which share a common vertex (called a clique-cut vertex) and/or (ii) cliques of order $\geq 3$ which are connected by an edge (a clique-cut edge) and/or (iii) cliques of order $\geq 3$ which are connected by a 3-path (end-vertices in the respective cliques) and/or (iv) cliques of order $\geq 3$ which are connected by a 4-path (endvertices in the respective cliques) and/or (v) cliques of order $\geq 3$ which are connected by a $k$-path, $k \geq 5$ (end-vertices in the respective cliques) and/or (vi) cliques of order $\geq 3$ with beams and/or (vii) pendent vertices.

For a tree $\mathrm{T} \not \mathrm{P}_{\mathrm{n}}, \mathrm{n} \geq 4$ and $\mathrm{T} \not \mathrm{K}_{1, \mathrm{~m}}, \mathrm{~m} \geq 3$ the line graph has a "tree-like" graphical embodiment i.e. besides cycles in cliques of order $\geq 3$, the line graph $\mathrm{L}(\mathrm{T})$ is acyclic. It typically has a combination of the structural elements (not necessarily all), (i) cliques of order $\geq 3$ which pairwise share a unique common vertex (called a clique-cut vertex) and/or (ii) cliques of order $\geq 3$ which are pairwise connected by an unique edge (a clique-cut edge) and/or (iii) cliques of order $\geq 3$ which are pairwise connected by an unique $k$-path, $k \geq 3$ (endvertices in the respective cliques and called a clique $k$-path) and/or (iv) cliques of order $\geq 3$ with beams such that, one beam is attached to one clique vertex (clique-beam) and/or (v) cliques of order $\geq 3$ with pendent vertices such that one pendent vertex is adjacent to one clique vertex (clique-pendent vertex).

Let a clique (or block) $Q_{n, i}$ of order $n \geq 2$ and $i \in \mathbb{N}$ an identifier be represented by a vertex $v_{Q_{n}, i}$. Derive the following graph from $L(T)$. Replace each clique $Q_{n, i}$ with a vertex $v_{Q_{n, i}}$ and add the edge between adjacent $v_{Q_{n, i}}$ and $v_{Q_{n, j}}$. If a clique $Q_{n, i}$ has only one cut vertex then add all non-cut vertices as leafs to $v_{Q_{n, i}}$. If a clique $Q_{n, j}$ has two or more cut vertices then delete all noncut vertices and all edges from $Q_{n, i}$. Clearly a tree results from this operation. This tree is called the confluence tree of $\mathrm{L}(\mathrm{T})$ and denoted by $\mho(\mathrm{L}(\mathrm{T}))$. 


\subsection{Heuristic B for minimum confluence set of line graph of tree}

For the line graph $\mathrm{L}(\mathrm{T}), \mathrm{T} \not \mathrm{P}_{\mathrm{n}}, \mathrm{n} \geq 4$ and $\mathrm{T} \not \mathrm{K}_{1, \mathrm{~m}}, \mathrm{~m} \geq 3$ do:

Step 1B. Construct the confluence tree $\mho(\mathrm{L}(\mathrm{G}))$ and relabel the vertices accordingly, $\mathfrak{u}_{1}, \mathfrak{u}_{2}, \mathfrak{u}_{3}, \ldots, \mathfrak{u}_{\mathrm{t}}$.

Step 2B. Apply Heuristic A to obtain $\zeta(\mho(L(T)))$

Step 3B. Yield $\zeta(\mathrm{L}(\mathrm{T}))=\zeta(\mho(\mathrm{L}(\mathrm{T})))$.

Theorem 12 Heuristic $B$ is valid.

Proof. The result for $\zeta\left(L\left(P_{n}\right)\right), n \geq 2$ is provided by Corollary 2 . Hence, the exclusion of paths is justified. The line graph $\mathrm{L}\left(\mathrm{K}_{1, \mathrm{~m}}\right), \mathrm{m} \geq 3$ is complete. Therefore the exclusion is justified.

Furthermore, in $\mathrm{L}(\mathrm{T})$ all 3-paths, if any, from a non-cut vertex of a clique must transverse through a cut vertex of the clique. Therefore, the vertex $v_{Q_{n, i}}$ represents all cut vertices in $Q_{n, i}$ definitively with regards to the definition of a confluence set. Therefore, Heuristic B is valid. Hence, $\zeta(\mathrm{L}(\mathrm{T}))=\zeta(\mho(\mathrm{L}(\mathrm{T})))$.

\section{$3.2 \quad$ Novel graph operations}

Two finite sets $\mathrm{X}$ and $\mathrm{Y}$ of equal cardinality is said to be identical if and only if $|X \cap Y|=|X|$, (or $|X \cap Y|=|Y|$ ). Otherwise the sets are distinct. Let $\mathbb{C}(\mathrm{G})=\{$ distinct minimum confluence sets of $\mathrm{G}\}$. Clearly, for a finite graph $\mathrm{G}$ the set $\mathbb{C}(G)$ exists and is finite. If a pendent vertex $u$ is attached to vertex $w$ of graph $\mathrm{G}$ the operation is denoted by $\mathrm{G}(\boldsymbol{w}) \multimap \mathfrak{u}$.

Theorem 13 For any graph $\mathrm{G}$ it follows that

$$
\zeta(\mathrm{G}) \leq \zeta(\mathrm{G}(w) \multimap \mathrm{u}) \leq \zeta(\mathrm{G})+1 .
$$

Proof. Consider $\mathrm{G}(\boldsymbol{w}) \multimap \mathrm{u}$. If a confluence set $\mathcal{C} \in \mathbb{C}(\mathrm{G})$ exists such that $\forall v \in \mathrm{V}(\mathrm{G})$ some shortest path $\mathrm{P}=v-\mathrm{u}_{(\mathrm{in} \mathrm{G}(w)-\mathfrak{o u})}$ exists such that $\mathrm{P}$ has a confluence vertex $x \in \mathrm{N}_{\mathrm{G}}[w]$ then $\zeta(\mathrm{G}(w) \multimap \mathfrak{u})=\zeta(\mathrm{G})$. If no such set exists then for any $\mathcal{C} \in \mathbb{C}(\mathrm{G})$ a set $\mathcal{C} \cup\{\boldsymbol{w}\}$ is a minimum confluence set of $\mathrm{G}(\boldsymbol{w}) \multimap \boldsymbol{u}$. Therefore, $\zeta(\mathrm{G}(w) \multimap \mathrm{u})=\zeta(\mathrm{G})+1$. Hence, $\zeta(\mathrm{G}) \leq \zeta(\mathrm{G}(w) \multimap \mathfrak{u}) \leq \zeta(\mathrm{G})+1$.

For graphs $G$ and $H$ let $u \in V(G), v \in V(H)$. By adding the edge $u v$ to connect graphs $G$ and $H$ the graph $G(u) \leadsto H(v)$ is obtained. The edge $u v$ is said to edge-merge graphs $\mathrm{G}$ and $\mathrm{H}$. Reversing the operation is simply called edge-unmerging or unmerging if the context is clear. 
Theorem 14 For $\mathrm{G}(\mathrm{u}) \leadsto \mathrm{H}(v)$ provided that $\mathrm{G} \neq \mathrm{K}_{1}$ and $\mathrm{H} \neq \mathrm{K}_{1}$ it follows that

$$
\zeta(\mathrm{G})+\zeta(\mathrm{H}) \leq \zeta(\mathrm{G}(\mathrm{u}) \leadsto \mathrm{H}(v)) \leq \zeta(\mathrm{G})+\zeta(\mathrm{H})+1 .
$$

Proof. (a) If a confluence set $\mathcal{C}_{1} \in \mathbb{C}(\mathrm{G})$ or $\mathcal{C}_{2} \in \mathbb{C}(\mathrm{H})$ exists such that $\mathrm{u} \in \mathcal{C}_{1}$ or $v \in \mathcal{C}_{2}$ then $\zeta(\mathrm{G}(\mathrm{u}) \leadsto \mathrm{H}(v))=\zeta(\mathrm{G})+\zeta(\mathrm{H})$.

(b) If in $\mathrm{G}$ and $\mathrm{H}$ respectively, $\mathrm{N}_{\mathrm{G}}(\mathrm{u}) \subseteq \mathcal{C}_{1}$ for some $\mathcal{C}_{1} \in \mathbb{C}(\mathrm{G})$ and $\mathrm{N}_{\mathrm{H}}(v) \subseteq \mathcal{C}_{2}$ for some $\mathcal{C}_{2} \in \mathbb{C}(\mathrm{H})$ then $\zeta(\mathrm{G}(\mathrm{u}) \leadsto \mathrm{H}(v))=\zeta(\mathrm{G})+\zeta(\mathrm{H})$.

(c) If both (a) and (b) fail it implies that $\forall \mathcal{C}_{1} \in \mathbb{C}(\mathrm{G})$ and $\forall \mathcal{C}_{2} \in \mathbb{C}(\mathrm{H})$ there exist vertices $x \in N_{G}(u), y \in N_{H}(v)$ such that the ( $\left.x, y\right)$-path (shortest) does not contain a confluence vertex of either $\mathrm{G}$ or $\mathrm{H}$. Therefore, and without loss of generality a set $\mathcal{C}_{1} \cup \mathcal{C}_{2} \cup\{u\}$ is a minimum confluence set of $\zeta(G(u) \leadsto H(v)$ ). The reasoning through (a), (b), (c) suffices to settle the result.

Definition 15 Let $S=\left(\mathrm{G}_{1}, \mathrm{G}_{2}, \mathrm{G}_{3}, \ldots, \mathrm{G}_{\ell}\right), \ell \geq 2$ be an ordered string of graphs. Each graph corresponds to order $n_{i}, 1 \leq i \leq \ell$ with corresponding vertex sets $\mathrm{V}\left(\mathrm{G}_{\mathrm{i}}\right)=\left\{v_{\mathrm{i}, \mathrm{j}}: 1 \leq \mathbf{j} \leq \mathrm{n}_{\mathbf{j}}\right\}$. For ordered pairs of vertices $\left(v_{\mathrm{t}, \mathrm{j}}, v_{\mathrm{t}+1, \mathrm{k}}\right)$, $1 \leq \mathrm{t} \leq \ell-1$ and $j \in\left\{1,2,3, \ldots, n_{\mathrm{t}}\right\}, \mathrm{k} \in\left\{1,2,3, \ldots, \mathrm{n}_{\mathrm{t}+1}\right\}$ the graph $\mathrm{G}_{\square \ell}=$ $\left(\left(\left(\mathrm{G}_{1} \odot \mathrm{G}_{2}\right) \sqcup \mathrm{G}_{3}\right) \cdots \odot \mathrm{G}_{\ell}\right)$ is obtained by merging each pair of ordered vertices $\left(v_{\mathrm{t}, \mathrm{j}}, v_{\mathrm{t}+1, \mathrm{k}}\right)$. This new graph is called a $\ell$-sliced graph.

Note that Definition 15 implies that all edges of the respective graphs $G_{i}$, $1 \leq i \leq \ell$ are retained. The merged vertex corresponding to say, $v_{t, j}$ and $v_{t+1, k}$ serves as a common end-vertex for all edges incident to $v_{\mathrm{t}, \mathrm{j}}$ and $v_{\mathrm{t}+1, \mathrm{k}}$. Reversing the merging operation is simply called vertex-unmerging or unmerging if the context is clear. By convention any graph $\mathrm{G}$ per se and in particular a graph without a cut vertex are said to be a 1-sliced graph.

Let $T$ be a tree on at least $n \geq 3$ vertices. Let $n=q+p$ with $q$ the number of pendent vertices. The next corollary is stated without proof as it is deemed to be self-evident.

Corollary 16 For any tree $\mathrm{T}$ of order $\mathrm{n} \geq 3$ (a lower bound for convenience) the line graph $\mathrm{L}(\mathrm{T})$ is a block graph which is a $\mathrm{p}$-sliced graph.

Theorem 17 Consider graph $\mathrm{G}$ of order $\mathrm{n}$ with at least one cut vertex. Unmerge sufficient cut vertices to obtain a maximum of $\mathrm{t}$ components. Then the graph $\mathrm{G}$ is a maximum $\mathrm{t}$-sliced graph.

Proof. Let $v$ be a cut vertex of $G$. Let the components of $G-v$ (cut-vertex deletion) be on vertices $V\left(G_{1}\right), V\left(G_{2}\right), \ldots V\left(G_{r}\right), r \geq 2$. Consider the induced 
subgraphs of $G$ i.e. $H_{1}=\left\langle V\left(G_{1}\right) \cup\{v\}\right\rangle, H_{2}=\left\langle V\left(G_{2}\right) \cup\{v\}\right\rangle, \ldots, H_{t}=\left\langle V\left(G_{r}\right) \cup\right.$ $\{v\}\rangle$. Clearly, in respect of $v \in \mathrm{V}\left(\mathrm{H}_{1}\right)$ and $v \in \mathrm{V}\left(\mathrm{H}_{2}\right)$ and $\ldots$, and $v \in \mathrm{V}\left(\mathrm{H}_{\mathrm{r}}\right)$ the graph obtained recursively, $\left(\left(\left(\mathrm{H}_{1} \bullet \mathrm{H}_{2}\right) \bullet \mathrm{H}_{3}\right) \cdots \bullet \mathrm{H}_{\mathrm{r}}\right)$ is isomorphic to $\cong \mathrm{G}$. In the case of removing $k \geq 2$ cut vertices to obtain the maximum $t$ components the result follows through immediate induction.

The notation $\mathrm{G}_{\square \ell}=\left(\left(\left(\mathrm{G}_{1} \sqcup \mathrm{G}_{2}\right) \sqcup \mathrm{G}_{3}\right) \cdots \bullet \mathrm{G}_{\ell}\right)$ per se does not specify the pairs of vertices under operation. Definition 13 requires that the pairs be specified prior. For application we consider only 2-sliced graphs in respect of $u \in V(G)$ and $v \in \mathrm{V}(\mathrm{H})$ denoted by, $\mathrm{G}(\mathrm{u}) \square \mathrm{H}(v)$. The result can be applied recursively to any $\ell$-sliced graph, $\ell \geq 3$.

Theorem 18 For graphs $\mathrm{G}$ and $\mathrm{H}$ and provided that $\mathrm{G} \neq \mathrm{K}_{1}$ and $\mathrm{H} \neq \mathrm{K}_{1}$ and; without loss of generality, $\mathrm{G} \neq \mathrm{K}_{2}$ and $\mathrm{H} \neq \mathrm{K}_{1}$ it follows that

$$
\zeta(\mathrm{G})+\zeta(\mathrm{H})-1 \leq \zeta(\mathrm{G}(\mathrm{u}) \bullet \mathrm{H}(v)) \leq \zeta(\mathrm{G})+\zeta(\mathrm{H})+1 .
$$

Proof. (a) If confluence sets $\mathcal{C}_{1} \in \mathbb{C}(\mathrm{G})$ and $\mathcal{C}_{2} \in \mathbb{C}(\mathrm{H})$ exist such that $\mathrm{u} \in \mathcal{C}_{1}$ and $v \in \mathcal{C}_{2}$ then $\zeta(\mathrm{G}(\mathrm{u}) \square \mathrm{H}(v))=\zeta(\mathrm{G})+\zeta(\mathrm{H})-1$.

(b) If a confluence set $\mathcal{C}_{1} \in \mathbb{C}(\mathrm{G})$ exists such that $u \in \mathcal{C}_{1}$ and $\nu \notin \mathcal{C}_{2} \forall \mathcal{C}_{2} \in \mathbb{C}(\mathrm{H})$ then $\zeta(\mathrm{G}(\mathrm{u}) \bullet \mathrm{H}(v))=\zeta(\mathrm{G})+\zeta(\mathrm{H})$.

(c) If in $\mathrm{G}$ and $\mathrm{H}$ respectively, $\mathrm{N}_{\mathrm{G}}(\mathrm{u}) \subseteq \mathcal{C}_{1}$ for some $\mathcal{C}_{1} \in \mathbb{C}(\mathrm{G})$ or $\mathrm{N}_{\mathrm{H}}(v) \subseteq \mathcal{C}_{2}$ for some $\mathcal{C}_{2} \in \mathbb{C}(\mathrm{H})$ then $\zeta(\mathrm{G}(\mathrm{u}) \square \mathrm{H}(v))=\zeta(\mathrm{G})+\zeta(\mathrm{H})$.

(d) If (a), (b) and (c) fail it implies that $\forall \mathcal{C}_{1} \in \mathbb{C}(\mathrm{G})$ and $\forall \mathcal{C}_{2} \in \mathbb{C}(\mathrm{H})$ there exist vertices $x \in N_{G}(u), y \in N_{H}(v)$ such that the $(x, y)$-path (shortest) does not contain a confluence vertex of either $\mathrm{G}$ or $\mathrm{H}$. Therefore, and without loss of generality a set $\mathcal{C}_{1} \cup \mathcal{C}_{2} \cup\{u\}$ is a minimum confluence set of $\zeta(G(u) \square H(v))$. Hence, $\zeta(\mathrm{G}(\mathrm{u}) \bullet \mathrm{H}(v))=\zeta(\mathrm{G})+\zeta(\mathrm{H})+1$.

The reasoning through (a), (b), (c) and (d) suffices to settle the result.

Note that Theorem 18 has an intriguing application to $K_{n} \square K_{m}, n \geq 2$, $\mathrm{m} \geq 2$. By convention $\zeta\left(K_{n}\right)=0, n \geq 1$. Hence, by convention the minimum confluence set $\mathcal{C}_{\mathrm{K}_{\mathrm{n}}}=\emptyset$. Therefore, Theorem 18(d) finds application.

\section{Cactus graphs}

For the purpose of studying cactus graphs (simply a cactus) it is noted that a cycle is meant to be a simple cycle or put differently, a chordless cycle. A cactus is a connected graph with at least one cycle and any two distinct cycle share at most one common vertex. Generally a cactus is denoted by $\mathrm{C}_{\mathrm{ac}}$. The cycle $\mathrm{C}_{3}$ is considered a trivial cactus. A well studied family of cactus graphs 
called the friendship graphs or $n$-fan graphs denoted by $F_{n}$ belongs to a wider family $\mathcal{F}=\left\{F^{(\mathfrak{n})}: F^{(\mathfrak{n})}\right.$. These graphs consist of $\mathfrak{n}$ cycles each of order $\geq 3$ which share a common vertex\}. Note that in a $F^{(n)}$ the respective order of distinct cycles may differ.

Proposition 19 For $\mathrm{F}^{(\mathrm{n})} \in \mathcal{F}$ and we have:

(a) $\zeta\left(\mathrm{F}^{(\mathrm{n})}\right)=1$ if $\mathrm{F}^{(\mathrm{n})}=\left(\left((\underbrace{\left.\left.\mathrm{C}_{3} \triangleright \mathrm{C}_{3}\right) \triangleright \mathrm{C}_{3}\right) \cdots \boxminus \mathrm{C}_{3}}_{\mathrm{n}-\text { copies }})\right.\right.$,

(b) $\zeta\left(\mathrm{F}^{(\mathrm{n})}\right)=\mathrm{n} \zeta\left(\mathrm{C}_{\mathrm{m}}\right)-(\mathrm{n}-1)$ if $\mathrm{F}^{(\mathrm{n})}=\left(\left((\underbrace{\left.\left.\mathrm{C}_{\mathrm{m}} \triangleright \mathrm{C}_{\mathrm{m}}\right) \bullet \mathrm{C}_{\mathrm{m}}\right) \cdots \boxminus \mathrm{C}_{\mathrm{m}}}_{\mathrm{n} \text {-copies }}), \mathrm{m} \geq 4\right.\right.$,

(c) $\zeta\left(\mathrm{F}^{(\mathrm{n})}\right)=\sum_{i=1}^{\mathrm{n}} \zeta\left(\mathrm{C}_{\mathrm{m}_{\mathrm{i}}}\right)-(\mathrm{n}-1)$ if $\mathrm{F}^{(\mathrm{n})}=\left(\left(\left(\mathrm{C}_{\mathrm{m}_{1}} \boxminus \mathrm{C}_{\mathrm{m}_{2}}\right) \boxminus \mathrm{C}_{\mathrm{m}_{3}}\right) \cdots \boxminus \mathrm{C}_{\mathrm{m}_{\mathrm{n}}}\right)$, $m_{i} \geq 4$,

(d) $\zeta\left(F^{(n)}\right)=\sum_{i=1}^{n-t} \zeta\left(C_{m_{i}}\right)-(n-t-1)$ if $F^{(n)}=\left(\left(\left(C_{m_{1}} \bullet C_{m_{2}}\right) \sqcup C_{m_{3}}\right) \cdots \boxminus C_{m_{n}}\right)$,

$\mathrm{m}_{\mathrm{i}} \geq 4$ for $1 \leq \mathrm{i} \leq \mathrm{n}-\mathrm{t}$ and $\mathrm{t}$ cycles are copies of $\mathrm{C}_{3}$.

Proof. (a) The proof will be by immediate induction. Consider two copies of $C_{3}$. Let first copy be on vertices $v_{1}, v_{2}, v_{3}$ and second copy on $\mathfrak{u}_{1}, \mathfrak{u}_{2}, \mathfrak{u}_{3}$. Without loss of generality let vertices $v_{1}, u_{1}$ merge as the common vertex in $\mathrm{C}_{3} \triangleright \mathrm{C}_{3}$. Label this common vertex as $\mathcal{w}_{1}$. Although $\zeta\left(\mathrm{C}_{3}\right)=0$ the existence of a 3-path say, $v_{2} w_{1} u_{2}$ necessitates $\mathcal{C}_{\mathrm{C}_{3}} \square \mathrm{C}_{3}=\left\{w_{1}\right\}$. By iteratively constructing $\left((\underbrace{\left.\left.C_{3} \square C_{3}\right) \square C_{3}\right) \cdots \square C_{3}}_{n \text {-copies }})\right.$ it follows through immediate induction that $\zeta\left(\mathrm{F}^{(\mathrm{n})}\right)=1$.

(b) Let the vertices of the $i^{\text {th }}$-copy of $C_{m}$ be labeled $v_{i, j}, j=1,2,3, \ldots, m$ and select a confluence set labeled $\mathcal{C}_{\mathrm{C}_{m}}^{\mathrm{i}}$ for each $1 \leq \mathfrak{i} \leq \mathfrak{n}$. Through stepwise rotation of the vertex labeling say, clockwise, it is possible to let $v_{1, i} \in \mathcal{C}_{\mathrm{C}_{\mathrm{m}}}^{i}, \forall i$. By merging vertices $v_{1,1}, v_{2,1}$ to yield $w_{1}$ in $C_{m} \vee C_{m}$ it follows that $\zeta\left(C_{m} \triangleright C_{m}\right)=$ $2 \zeta\left(C_{m}\right)-1=2 \zeta\left(C_{m}\right)-(2-1)$. After following similar iterative procedure the result $\zeta\left(F^{(n)}\right)=n \zeta\left(C_{m}\right)-(n-1)$ follows through immediate induction.

(c), (d). These results follow through similar reasoning found in parts (a) and (b).

Note that in the first iteration in the proof of Proposition 19(a), Theorem 18(d) applies. In the induction step, Theorem 18(b) applies. In the proofs of parts (b) through (d) Theorem 18(a) applies.

Essentially $\mathrm{G} \odot \mathrm{H}$ means that graphs $\mathrm{G}$ and $\mathrm{H}$ are connected by merging two vertices $v \in V(G)$ and $u \in V(H)$ which results in a cut vertex say $w$. The term unmerging $w$ means to disconnect $\mathrm{G}$ and $\mathrm{H}$ by cloning $w$ into say, 
$v \in V(G)$ and $u \in V(H)$. Furthermore, if a combination of more than three cycles and/or trees share a common vertex $w$ then unmerging $w$ may occur for graphical subsets. It means that if say tree $T$ and cycles $C_{n}, C_{m}$ share vertex $w$, the graphical subsets say $\{T\},\left\{C_{n}, C_{m}\right\}$ can unmerge to render $C_{n} \boxminus C_{m}$ with common vertex $w$ and T unmerges with new vertex say $v$ substituting $w$. Consider a cactus. Besides the wider meaning of connectivity we specialize to say, two connected cycles will mean directly connected through either a common vertex (cut vertex) or a common edge (cut edge). If a cycle is connected to a tree it means directly connected to a maximal tree. Hence, the maximal tree and the cycle only share a common vertex. Two cycles connected by an edge may only unmerge by edge deletion. The next corollary is deemed to be self-evident.

Corollary 20 Any cactus $\mathrm{C}_{\mathrm{ac}}$ can be unmerged into a minimum number of maximal graphical structures i.e. cycles, maximal trees and edges.

Theorem 21 (Existence theorem) There exists a heuristic algorithm to find both the confluence number and a minimum confluence set of a cactus.

Proof. It is known from [11] that finding a minimum confluence set of a graph $\mathrm{G}$ is NP-complete. Iterative pairwise unmerging of a cactus $\mathrm{C}_{\mathrm{ac}}$ in an arbitrary order to find a minimum number of maximal graphical structures i.e. cycles, maximal trees and edges is possible. Pairwise unmerging is possible in a finite number of steps and the solution (cluster of graphical structures) is unique. Let the arbitrary ordered pairwise unmerging steps be $s_{1}, s_{2}, \ldots, s_{\ell}$. Furthermore, an ordered set of prescriptions exist to orderly reconstruct the cactus through pairwise merging. The prescriptions are in the order $s_{\ell}^{-1}, s_{\ell-1}^{-1}, \ldots, s_{1}^{-1}$. Therefore, the original cactus is uniquely reconstructed. Let a merging operation be $f \in\{\boxminus, \leftrightarrow \rightsquigarrow\}$.

Step $s_{\ell}^{-1}$. Label the one graphical structure $G_{1}$ and the other $H_{1}$. Apply $s_{\ell}^{-1}=G_{1} f_{1}$ to obtain $G_{2}$. Depending on $f, G_{1}, H_{1}$ any one of Theorems 14 or 18 ensures that a minimal confluence set and the confluence number of $\mathrm{G}_{2}$ can be found.

Step $s_{\ell-1}^{-1}$. Consider $G_{2}$ and the appropriate graphical structure $\mathrm{H}_{2}$ prescribed by $s_{\ell-1}^{-1}$. Apply $s_{\ell-1}^{-1}=G_{2} f H_{2}$ to obtain $G_{3}$. Depending on $f, G_{2}, H_{2}$ any one of Theorems 14 or 18 ensures that a minimal confluence set and the confluence number of $\mathrm{G}_{3}$ can be found.

Through immediate induction it follows that through inversing the merges, $s_{\ell}^{-1}, s_{\ell-1}^{-1}, \ldots, s_{1}^{-1}$ the confluence number and a minimum confluence set of a cactus can be found. Thus a heuristic algorithm exists. 


\section{$5 \quad$ Linear Jaco graphs}

Linear Jaco graphs was introduced by Kok et.al, [5, 6, 7, 9]. Linear Jaco graphs are digraphs. Therefore the definitions define arcs. It is important to note that in the notation $J_{\mathfrak{n}}(f(x))$ means that a Jaco graph is of order $n$ and for vertex $v_{i}$ the vertex degree is bounded by $\operatorname{deg}\left(v_{i}\right) \leq f(i)$. For the family of linear Jaco graphs $f(x)$ is restricted to linear functions. Reference to the definitions will assist in some proofs to follow. For ease of reference we recall the formal definitions.

Definition 22 The infinite linear Jaco graph $\mathrm{J}_{\infty}(\mathrm{x}), \mathrm{x} \in \mathbb{N}$ is defined by $\mathrm{V}\left(\mathrm{J}_{\infty}(\mathrm{x})\right)=\left\{v_{\mathrm{i}}: i \in \mathbb{N}\right\}, \mathrm{A}\left(\mathrm{J}_{\infty}(\mathrm{x})\right) \subseteq\left\{\left(v_{i}, v_{j}\right): i, j \in \mathbb{N}, i<j\right\}$ and $\left(v_{i}, v_{j}\right) \in$ $\mathrm{A}\left(\mathrm{J}_{\infty}(\mathrm{x})\right)$ if and only if $2 \mathrm{i}-\mathrm{d}^{-}\left(v_{\mathrm{i}}\right) \geq \mathrm{j}$.

Definition 23 The family of finite linear Jaco graphs is defined by $\left\{\mathrm{J}_{\mathrm{n}}(\mathrm{x}) \subseteq\right.$ $\left.\mathrm{J}_{\infty}(\mathrm{x}): \mathrm{n}, \mathrm{x} \in \mathbb{N}\right\}$. A member of the family is referred to as the Jaco graph, $\mathrm{J}_{\mathrm{n}}(\mathrm{x})$.

We shall only consider the underlying graph (undirected). See figure 2. However, within the context the notions of in-degree $\operatorname{deg}^{-}\left(v_{i}\right)$ and out-degree $\operatorname{deg}^{+}\left(v_{i}\right)$ remains relevant. Since the linear Jaco graphs for $n=1,2,3,4$ are $K_{1}, K_{2}, P_{3}, P_{4}$ respectively. Because $d^{+}\left(v_{3}\right)=2 \geq 2$ the lower bound $n \geq 3$ will be considered.

For $\mathrm{J}_{5}(\mathrm{x})$ vertex $v_{3}$ is a confluence vertex therefore $\mathcal{C}_{\mathrm{J}_{5}(\mathrm{x})}=\left\{v_{3}\right\}$. To constructively find a minimum confluence set of $J_{n}(x), n \geq 3$ we call vertex $v_{3}$ the prime confluence vertex of all linear Jaco graphs. When we skip or hop from say vertex $v_{i}$ to $v_{j}$ (not necessarily adjacent) it is denoted by $v_{i \curvearrowright j}=v_{j}$. For example, let $\ell(i)=(i+2)+\operatorname{deg}^{+}\left(v_{i+1}\right)$. Then $v_{3 \curvearrowright \ell(3)}=v_{8}$ and $v_{8 \curvearrowright \ell(8)}=v_{16}$. See table 1 , page 71 in [5]. Let an $j^{\text {th }}$-iteration yielding a minimum confluence set be denoted by $i t_{j}$. Define $i t_{1} \longmapsto\left\{v_{3}\right\}$, it $t_{2} \longmapsto\left\{v_{3}, v_{8}\right\}$ and $i t_{j+1} \longmapsto$

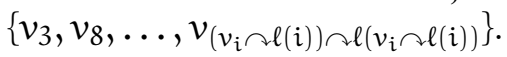

Lemma 24 For a Jaco graph $\mathrm{J}_{\mathrm{n}}(\mathrm{x}), \mathrm{n} \geq 3$ a confluence set is given by $X=\left\{v_{3}, v_{8}, v_{8 \curvearrowright \ell(8)}, v_{16 \curvearrowright \ell(16)}, \ldots, v_{t}=v_{\left(v_{i} \curvearrowright \ell(i)\right) \curvearrowright \ell\left(v_{i} \curvearrowright \ell(i)\right)}\right\}, t \leq n$.

Proof. The result may be said to be trivial because for any vertex $v_{i} \in X$, all vertices $v_{j},(i-2)-\operatorname{deg}^{-}\left(v_{i-1}\right) \leq j \leq \ell(i)$ have $\mathrm{d}\left(v_{i}, v_{j}\right) \leq 2$. Hence, by the definition of a confluence set, $X$ is a confluence set of $J_{n}(X)$.

The induced path $\left\langle\left\{v_{1}, v_{2}\right\} \cup X\right\rangle$ is called the confluence path of $\mathrm{J}_{\mathfrak{n}}(\mathrm{x})$. It is denoted by $\mho_{\mathrm{P}}\left(\mathrm{J}_{\mathrm{n}}(\mathrm{x})\right)$. 


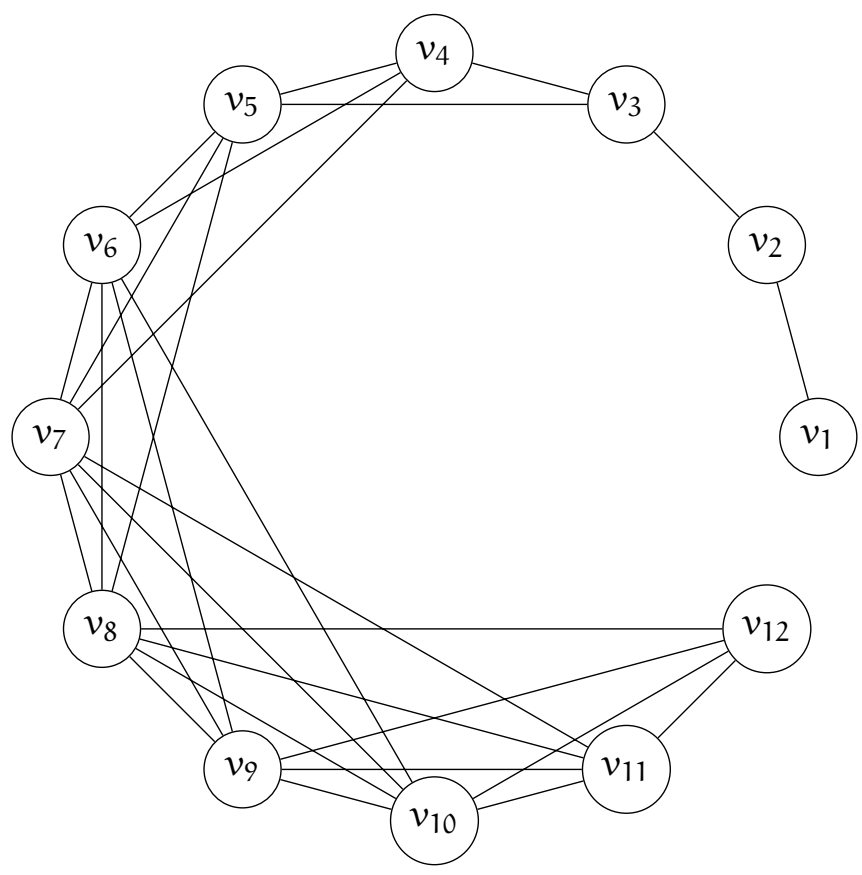

Figure 2: Linear Jaco graph $\mathrm{J}_{12}(\mathrm{x})$.

Theorem 25 For $n \geq 3, \zeta\left(J_{n}(x)\right)=\zeta\left(\mho_{\mathrm{P}}\left(J_{n}(x)\right)\right)$.

Proof. From Lemma 24, $\zeta\left(J_{n}(x)\right) \leq|X|$. From Definition 22 it follows if $X^{\prime}=$ $X \backslash\left\{v_{j}\right\}, v_{i} \in X$ then some vertices $v_{k}, k<i$ and $v_{t}, t>i$ exist with $\mathrm{d}\left(v_{k}, v_{t}\right) \geq 3$. Therefore $\zeta\left(J_{n}(x)\right)>\left|X^{\prime}\right|$. Hence, $\zeta\left(J_{n}(x)\right)=|X|$.

Define sub-families of linear Jaco graphs as follows, $\mathcal{F}_{1}$ on $n_{1,1}=3 \leq n \leq 7=$ $n_{1,2}$ vertices, $\mathcal{F}_{2}$ on $n_{2,1}=8 \leq n \leq 15=n_{2,2}$ vertices, $\mathcal{F}_{3}$ on $n_{3,1}=\ell(8) \leq$ $\mathrm{n} \leq \ell(9)-1=n_{3,2}$ vertices, $\ldots, \mathcal{F}_{i}$ on $n_{i, 1}=\ell\left(n_{i-1,1}\right) \leq n \leq \ell\left(n_{i, 1}\right)-1=n_{i, 2}$ vertices.

Corollary 26 For $n \geq 3$ let $\mathrm{J}_{\mathfrak{n}}(\mathrm{x}) \in \mathcal{F}_{\mathfrak{i}}$ then $\zeta\left(\mathrm{J}_{\mathfrak{n}}(\mathrm{x})\right)=i$.

Proof. The results follow by similar reasoning found in the proof of Lemma 24.

Corollary 27 For $\mathrm{J}_{\mathfrak{n}}(x), \mathfrak{n} \geq 3$, Let the diameter of $\mathrm{J}_{\mathfrak{n}}(x)$ be $\operatorname{diam}\left(\mathrm{J}_{\mathfrak{n}}(x)\right)=$ k. Then $\zeta\left(J_{n}(x)\right)=\left\lfloor\frac{k+1}{3}\right\rfloor$.

Proof. From Definition 22 it follows that if vertices $v_{i}, v_{j}, i<j$ are adjacent then $v_{k}, v_{l}, i \leq k<l \leq j$ are adjacent. There exists a path $\mathrm{P}_{k+1}$ (of length 
k) such that any $v_{i} \in \mathrm{V}\left(\mathrm{J}_{\mathfrak{n}}(\mathrm{x})\right.$ and any vertex $v_{j}$ on $\mathrm{P}_{k+1}$ has $\mathrm{d}\left(v_{i}, v_{j}\right) \leq 2$. Therefore path $P_{k+1}$ is a path of $\max \{\min \}$ length in $J_{n}(x)$. The result finally follows from the result for paths.

To illustrate reasoning in the proof of Corollary 27 see figure 2 . Since edge $v_{6} v_{10}$ exists all pairs of vertices in $\left\{v_{6}, v_{7}, v_{8}, v_{9}, v_{10}\right\}$ are adjacent. Put differently, the induced subgraph $\left\langle\left\{v_{6}, v_{7}, v_{8}, v_{9}, v_{10}\right\}\right\rangle \cong \mathrm{K}_{5}$.

\section{Conclusion}

Theorem 4 characterized paths which have unique minimum confluence sets. Based on this characterization a few research avenues are highlighted.

Problem 1. Characterize graphs with unique minimum confluence sets.

Solving Problem 1 opens an avenue to characterize forbidden graphs in relation to confluence number and minimum confluence set. The next conjecture could be the key.

Conjecture 1. Let $\zeta(G) \geq 2$. A minimum confluence set $\mathcal{C}_{\mathrm{G}}$ of $\mathrm{G}$ has, for each confluence vertex $u \in \mathcal{C}_{\mathrm{G}}$ at least one vertex $v_{\neq u} \in \mathcal{C}_{\mathrm{G}}$ such that the distance $\mathrm{d}_{\mathrm{G}}(\mathrm{u}, v) \leq 3$.

When a vertex $u \in V(G)$ is selected whereupon a set $X$ which complies with some parameter set prescriptions and $u \in X$ it is said that, $X$ has been built on $u$. If Conjecture 2 is found to be correct the next conjecture with motivation might be settled.

Conjecture 2. Let $\mathrm{G}$ be a non-complete connected graph of order $n \geq 3$ and $G$ does not have a unique minimum confluence set. There exists a minimum confluence set $\mathcal{C}$ of $\mathrm{G}$ which contains a vertex $u$ with $\operatorname{deg}_{\mathrm{G}}(\mathrm{u})=\Delta(\mathrm{G})$.

Motivation: Firstly, it is possible to select a vertex $u$ and "build" a minimal confluence set $X$ for $G$. It is claimed that amongst all vertices with $\operatorname{deg}_{\mathrm{G}}(v)=\Delta(\mathrm{G})$ at least one such "built" $X^{\prime}$ on say $v^{\prime}$ will be a minimum confluence set because firstly, there exists a $v^{\prime}$ such that $\left|X^{\prime}\right|=\min \{X$ : built on $\mathfrak{u}$, $\left.\operatorname{deg}_{\mathrm{G}}(\mathrm{u})=\Delta(\mathrm{G})\right\}$ and $\left|\mathrm{N}\left(v^{\prime}\right)\right|=\Delta(\mathrm{G})$ contributes a maximum to $\mathrm{Y}$ with $\mathrm{Y}=\bigcup_{w \in X^{\prime}} \mathrm{N}[w]$.

It is easy to verify the result for the one graph order 3, the five graphs of order 4, the twenty graphs of order 5 all of which are non-complete connected graphs. Assume the result holds for all non-complete connected graphs of order $k$. Consider any non-complete connected graph $G$ of order $k+1$. Amongst all vertices with degree $\delta(G)$ select a vertex $w$ with minimum $\left|N_{2}(w)\right|$ such that $w$ is not a cut-vertex. Such $w$ always exists. Consider graph $\mathrm{H}=\mathrm{G}-w$. 
Since $\mathrm{H}$ is a non-complete connected graph of order $\mathrm{k}$ consider a minimum confluence set $\mathcal{C}_{\mathrm{H}}$ which contains a vertex $x$ with $\operatorname{deg}_{\mathrm{H}}(\mathrm{x})=\Delta(\mathrm{H})$.

Case 1. If $\operatorname{deg}_{H}(x)=\operatorname{deg}_{G}(x)-1$ then $w$ is adjacent to $x$ in $G$ and the result holds.

Case 2. If $\operatorname{deg}_{H}(x)=\operatorname{deg}_{G}(x)$ then $w$ is not adjacent to $x$ in $G$. If $w$ is adjacent to a vertex in $\mathrm{N}[\mathrm{y}], \mathrm{y} \in \mathcal{C}_{\mathrm{H}}$ the result holds for $\mathrm{G}$. If $w$ is adjacent to a vertex $\mathrm{t} \in \mathrm{N}_{2}[\mathrm{y}], \mathrm{y} \in \mathcal{C}_{\mathrm{H}}$ then $\mathrm{X}^{\prime}=\mathcal{C}_{\mathrm{H}} \cup\{\mathrm{t}\}$ is necessarily a minimum confluence set of $G$. Therefore, the results holds for $G$ of order $k+1$. Hence, $\zeta(G)=\left|X^{\prime}\right|$.

By induction the result holds for all non-complete connected graphs $G$ be of order $n \geq 3$. $\quad \square$ Following Theorem 21 it is important to develop and code an efficient algorithm to further research for graphs in general. Computational research is an avenue to to find substantial results for the new notion. The three novel graph operations with the results of Theorems 13, 14, $17 \& 18$ provide a framework to achieve an algorithmic approach.

Problem 2. Design and code an efficient algorithm to find a minimum confluence set an the confluence number of a graph.

For the construction of $\mho(L(T))$ the pseudo entities, representative vertices for cliques were utilized. Upon utilizing Heuristic A the procedure remained blind to the pseudo entities. Such representative vertices may appear in the minimum confluence set yielded by Heuristic A. Although $\zeta(\mathrm{L}(\mathrm{T}))$ is valid same cannot be said of the set $\mathcal{C}_{\mathrm{L}(\mathrm{T})}$.

Problem 3. If possible find an improved construction of a confluence tree for the line graph of a tree which will yield both the confluence number and a valid minimum confluence set.

If some blocks of a block graph are substituted by general graphs a blocklike graph $\mathrm{G}$ is constructed. It implies that $\mathrm{G}$ can be decomposed into a maximum number of graphs through unmerging operations $f^{-1}, f \in\{\square, \leftrightarrow,-\longrightarrow\}$. Let such decomposition result in a set of minimal subgraphs denoted by $\mathcal{D}(\mathrm{G})=\left\{\mathrm{G}_{1}, \mathrm{G}_{2}, \mathrm{G}_{3}, \ldots, \mathrm{G}_{\mathrm{t}}\right\}$. Let $\mathrm{S}=\left(\zeta\left(\mathrm{G}_{1}\right), \zeta\left(\mathrm{G}_{2}\right), \zeta\left(\mathrm{G}_{3}\right), \ldots, \zeta\left(\mathrm{G}_{\mathrm{t}}\right)\right)$. Iteratively reconstructing $G$ with the results of Theorems 13,14 and 18 will determine $\zeta(G)$. This avenue of research remains open.

Let $\zeta(G)=k$. For any minimum confluence $\mathcal{C}_{\mathrm{G}}$ let $\mathrm{V}^{\prime}(\mathrm{G})=\mathrm{V}(\mathrm{G}) \backslash \mathcal{C}_{\mathrm{G}}$. Let $Z=\left\{Z_{i}: Z_{j}\right.$ is a $k$-tuple subset of vertices of $\left.V^{\prime}(G)\right\}$.

Conjecture 3. There exist a minimum confluence set $\mathcal{C}^{\prime}$ of $\mathrm{G}$ such that,

$$
\sum_{v \in \mathcal{C}^{\prime}} \operatorname{deg}_{G}(v) \geq \sum_{u \in Z_{i}, \forall Z_{i} \in Z} \operatorname{deg}_{G}(u) .
$$


In the field of algebra it is known that posets of minimal type are related to linear Jaco graphs $[1,8]$. Section 5 suggests that algebraic results may follow in respect of the minimum confluence number and confluence path of linear Jaco graphs. It is deemed a worthy avenue for research.

\section{Dedication}

This paper is dedicated to the life and career of the first author's remarkable Uncle Ben Kok. Uncle Ben was born on 18 December 1930 and at the time of writing this paper, he continues to show a keen interest in the advances in mathematics, quantum physics, nuclear physics and modern day technology. Linear Jaco graphs were named after Pieter Jaco Kok, an elder brother of Uncle Ben Kok. The dedication is to celebrate the occasion of Uncle Ben's $90^{\text {th }}$ birthday.

\section{Acknowledgment}

The authors would like to thank the anonymous referees for their constructive comments, which helped to improve on the elegance of this paper.

\section{References}

[1] R. Assous, M. Pouzet, Jónsson posets, Algebra Universalis 79, 3 (2018) article 74. $\Rightarrow 37$

[2] A. Behtoei, M. Jannesari, B. Taeri, A characterization of block graphs, Discrete Applied Mathematics 158 (2010) 219-221. $\Rightarrow 27$

[3] J.A. Bondy, U.S.R. Murty, Graph Theory with Applications, Macmillan Press, London, $1976 . \Rightarrow 21$

[4] F. Harary, Graph Theory, Addison-Wesley, Reading MA, 1969. $\Rightarrow 21$

[5] J. Kok, Linear Jaco graphs: A critical review, Journal of Informatics and Mathematical Sciences 8, 2 (2016) 67-103. $\Rightarrow 33$

[6] J. Kok, P. Fisher, B. Wilkens, M. Mabula, V. Mukungunugwa, Characteristics of finite Jaco graphs, $\mathrm{J}_{\mathfrak{n}}(1), \mathrm{n} \in \mathbb{N}$, arXiv: $1404.0484 v 1$ [math.CO]. $\Rightarrow 33$

[7] J. Kok, P. Fisher, B. Wilkens, M. Mabula, V. Mukungunugwa, Characteristics of Jaco graphs, $\mathrm{J}_{\infty}(\mathrm{a}), \mathrm{a} \in \mathbb{N}$, arXiv: $1404.1714 v 1$ [math.CO]. $\Rightarrow 33$

[8] J. Kok, N.K. Sudev, K.P. Chithra, U. Mary, Jaco-type graphs and black energy dissipation, Advances in Pure and Applied Mathematics 8, 2 (2017) 141-152. $\Rightarrow$ 37 
[9] J. Kok, C. Susanth, S.J. Kalayathankal, A study on linear Jaco graphs, Journal of Informatics and Mathematical Sciences 7, 2 (2015) 69-80. $\Rightarrow 33$

[10] A.C.M. Rooij, H.S. Wilf, The interchange graph of a finite graph, Acta Mathematica Hungarica 16, 3-4 (1965) 263-269. $\Rightarrow 22,23$

[11] J. Shiny, J. Kok, V. Ajitha, Confluence number of graphs, Communicated. $\Rightarrow$ $21,22,23,32$

[12] B. West, Introduction to Graph Theory, Prentice-Hall, Upper Saddle River, 1996. $\Rightarrow 21$

Received: December 18, 2020 • Revised: January 31, 2021 